\title{
Cavitation shapes measurements in piston-ring lubrication and their link to lubricant properties
}

\author{
Polychronis Dellis ${ }^{1 *}$ \\ ${ }^{1}$ School of Mechanical Engineering Educators, Assistant Professor - Scientific Associate, ASPETE, \\ Heraklion, Attiki, 14121, Athens
}

\begin{abstract}
The emissions control regulations introduced by governments are set to improve engine quality and reduce the impact automobiles have on the planet. The regulations imposed on the manufactures have proven very difficult to meet. To this effect some of the leading names in the industry were pushed to invest significant funding in research, development and optimisation of combustion, powertrain and tribology inside the ICE. Their goal is reduction of fuel consumption and emissions while increasing performance and durability. The piston-ring and cylinder-liner interaction is the major source of frictional losses for reciprocating ICEs and so, it is important to avoid any failure of piston-rings to effectively control lubricant transport from the sump onto the cylinder walls and further to the combustion chamber. This lubricant will participate in the emissions through absorption and desorption of fuel in the oil film at the cylinder walls, also resulting in lubricant contamination and consumption. The objective of this project is to assist with the investigation of phenomena occurring in the cylinder liner and piston-ring interaction under different operating conditions. The following investigations have been carried out, flow and cavitation visualisation in a model lubricant rig and cavitation visualisation in a newly designed optical engine.
\end{abstract}

\section{Introduction}

Lubricant flow in between and under the piston-rings and liner of the internal combustion engine has been a challenging study subject because of its complexity in the real engine and many factors that contribute or obstruct the effective, low-emission and energy efficient operation.

To such extent previous studies have been accomplished with a focus on simplifying lubrication at the interface together with simulation of the theoretical equations that accompany lubricant flow $[1,2,3,4,5,6,7,8,9,10]$. A single ring simulating test rig provides the mechanism with the necessary measurement options to contribute to this study $[2,3,4,5]$. Further on, engine applications in motored and firing engine have been conducted to assess the lubricant flow and compare the simplification study to the real engine results [5, $6,9]$.

Along with friction, oil film thickness - both with laser induced fluorescence (LIF) sensors and capacitance, pressure measurements and visualization - that provided robust and

\footnotetext{
*Corresponding Author, pasd@city.ac.uk
} 
repeatable results, different forms of cavitation have been identified within these various measurements $[2,7,8]$ for the case of the single-ring test rig, that affect lubricant flow. The formation of cavities and their subsequent disposition affect the pressure generated in the continuous thin lubricant film, and hence, the load capacity.

Valuable information of lubricant flow properties under the specified circumstances are gathered which, in turn, lead to understanding of the flow in an ordinary engine piston-ring pack and eventually to characterisation of the lubricant transport phenomena in the pistonring pack of production engines $[5,6]$ along with a link, via a parametric study, to the effect of lubricant properties in oil film characteristics $[1,11,12]$.

It is therefore important to study cavitation as it can cause serious damage to fluid handling machinery [8]. Along with cavitation shapes study in comparison to the overall piston width, the effect of oil properties and their subsequent influence on cavitation (large cavitation area at the interface of the ring-liner assembly or cavity-shape details regarding the size (width or length) of the cavitation forms) at oil film rupture should also be taken into account.

Video images captured with two high speed cameras coupled with three high intensity light sources were analysed. The images were post-processed with the aid of a custom build algorithm. The basic function of this algorithm is the extraction of matrices with elements of cavity length, cavity width, area of cavitation and number of cavities present under the pistonring and the cylinder liner [5]. Visualisation experiments are a quick, efficient and effective way to test different lubricant samples and compare their performance in terms of physical, chemical properties and cavitation initiation and development throughout the stroke. This parametric study offered valuable results enabling the performance of each lubricant to be assessed. Direct link between the lubricant formulation and the operating conditions could be established.

\section{Experimental Set-up}

The study was conducted in three experimental test rigs. Two optical engines were used, with their cylinder block purpose built to visualise cavitation through image acquisition and processing and a single-ring test rig that simulates the piston-liner movement but the motion is inversed, i.e., it consists of a steady $5 \mathrm{~mm}$ width piston-ring section fixed under a flat liner moving section. Two single cylinder engines were used, a Lister-Petter PHW1 diesel engine used only for motoring experiments with its liner as well as the cylinder block modified to fit glass window sections for viewing purposes [3] and a Ricardo HYDRA engine, again modified to accommodate larger viewing sections [5].

\subsection{Single-Ring Test Rig Sensors and Optical Engines Set-up}

With the use of the single-ring test rig itself, valuable information about piston-ring lubrication under simplified conditions were acquired. The ring-liner interface was easy to access and work with and so, a series of lubricant parametric measurements from the fitted sensors were post-processed with the advantage of eliminating the engine in-cylinder complications resulting from its inherent complexity (ring dynamics, thermal and elastic deformation of the rings, starved lubrication, circumferential ring variations, lubricant degradation and blow-by) [8].

Oil film thickness is measured both by a capacitance sensor (electrical method) fixed next to the piston-ring specimen (inside the oil bath or outside) an LIF (laser induced fluorescence) sensor fixed in the liner body, while the sensor itself is flush with its surface under examination to avoid any interruption in the development of the lubricant film; and a 
pressure sensor that combined with the visualisation data provides valuable oil film pressure measurements that compliment cavitation visualisation data. Friction is measured via a force measurement sensor mounted outside the oil bath. The optical liner consists of a glass section aided in studying further cavitation. Initial evidence of cavitation was taken from the LIF measurements. In Figure 1a) a schematic of the liner is shown and in Figure 1b) a photo the piston-ring section set-up for the experiments, the oil jets, the friction and the capacitance sensor. In Figure 1c) the Lister-Petter engine can be seen with the modified sections of liner and block.

The single cylinder Lister-Petter PHW1 engine was used for visualisation imaging in motoring tests. This engine had its block and liner modified to accommodate a quartz window for the experiments. Results from engine testing could be compared to the test rig results, concerning the initiation and development of cavitation [11].

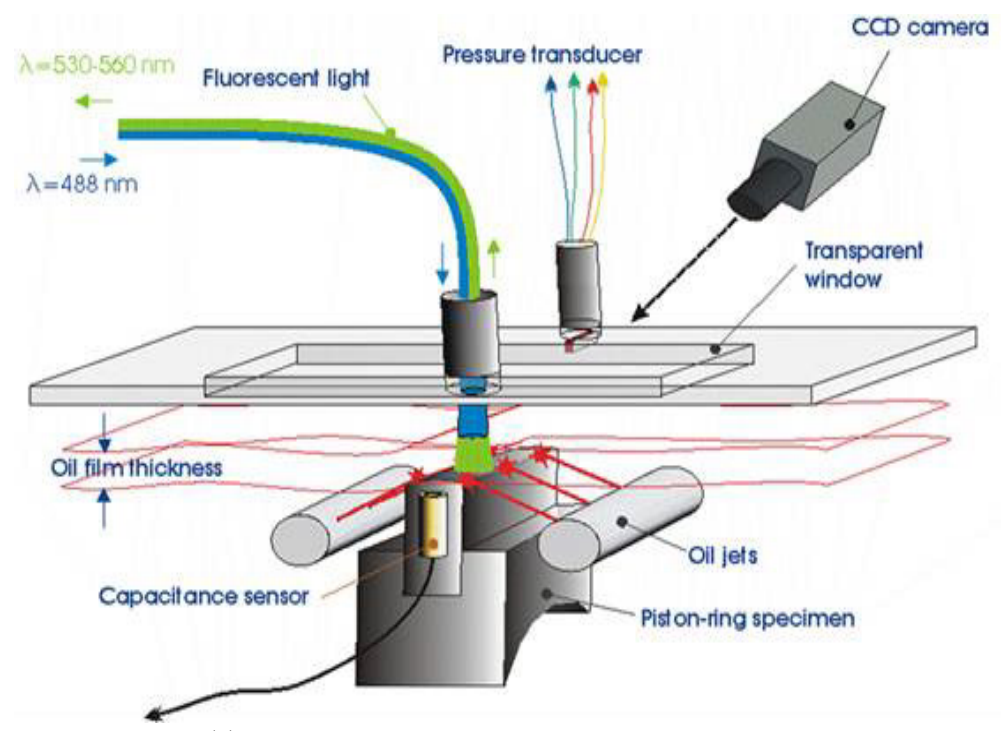

(a)

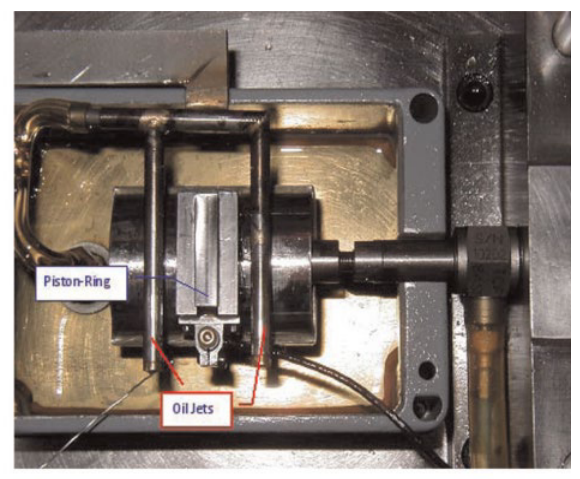

(b)

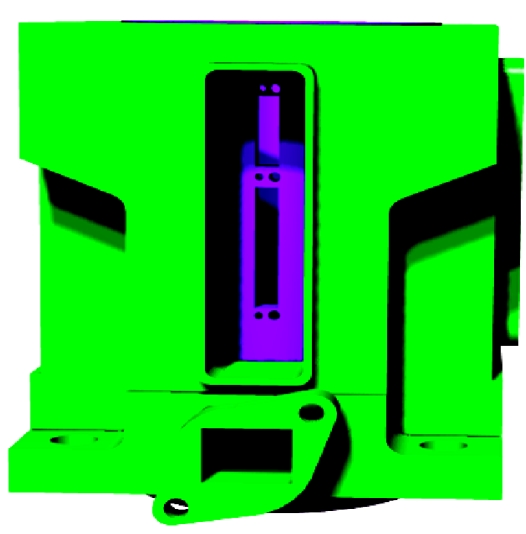

c)

Fig. 1. a) The single ring test rig with the measurement sensors: i) capacitance sensor, ii) LIF sensor both for oil film thickness measurements, iii) glass section for visualisation, iv) miniature pressure transducer and b) Close-up of the piston-ring specimen with the friction sensor shown c) drawing of the Lister-Petter engine block and liner modifications [7]. 


\subsubsection{Single-ring test rig and Lister-Petter engine results}

In previous studies the evaluation of pressure measurements and visualisation tests showed clear forms of cavitation at the interface between the piston-ring and the liner surface [2, 4, $7,9,11]$. In Figure 2 the shapes that have been used for evaluation can clearly be seen [5]: In these images, taken at different parts of the stroke, an estimation of the area of cavitation was further processed for the case of the single ring test rig. That includes measurements of the length of cavities captured in the images, the number of cavities in the upper and lower part of the captured image and the total area covered in the same image. A custom-built algorithm was used to process the image data, based on the contrast scale of each image.

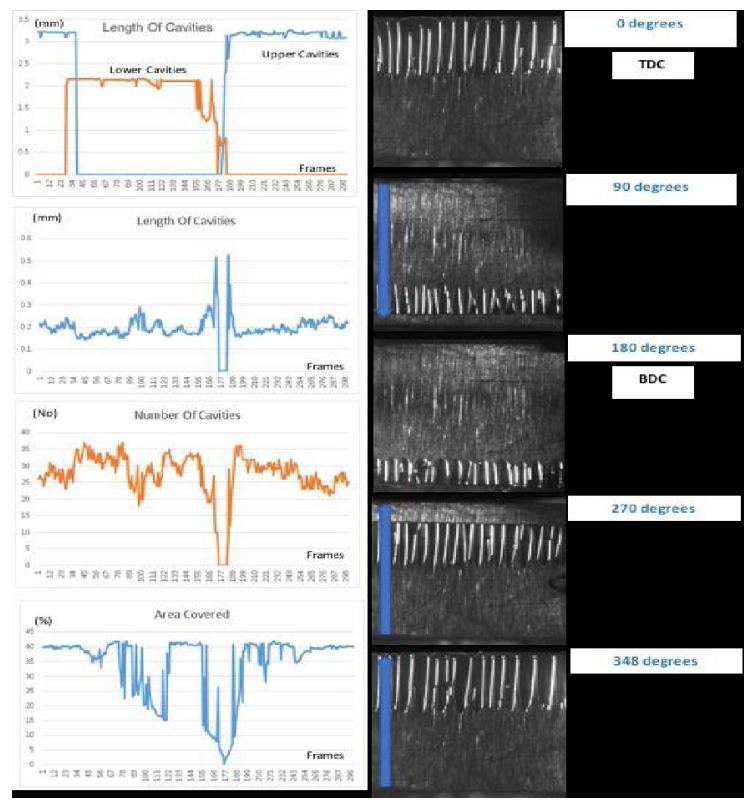

Fig. 2. Cavitation images from the test rig and the algorithm results [5]

In the past, it was suggested that a cavitation number is introduced $[7,11]$ so that imaging from cavitation can be quantified. This coefficient is the ratio of the width or the length of the string cavities divided to the piston ring width.

$$
\text { Cavitation } \text { factor }_{i}=\frac{\text { width of string }}{\text { width of piston ring }}
$$

Cavitation factors (CF) calculated for the engine LISTER-PETTER engine $\mathrm{CF}_{\text {engine }}=$ 0.194 and respectively for the test rig $\mathrm{CF}_{\text {test rig }}=0.054 . \mathrm{CF}_{\text {engine }}$ is $72.16 \%$ greater than $\mathrm{CF}_{\text {test }}$ rig. In the case of the single ring test rig, the strings are covering approximately $50 \%$ of the piston ring length. In terms of their shape, they are very uniform. In Figure 3 the cavitation shapes of the Lister Petter engine can be viewed and the cavitation factor has been extrapolated from shape measurements on the images shown [11]. 


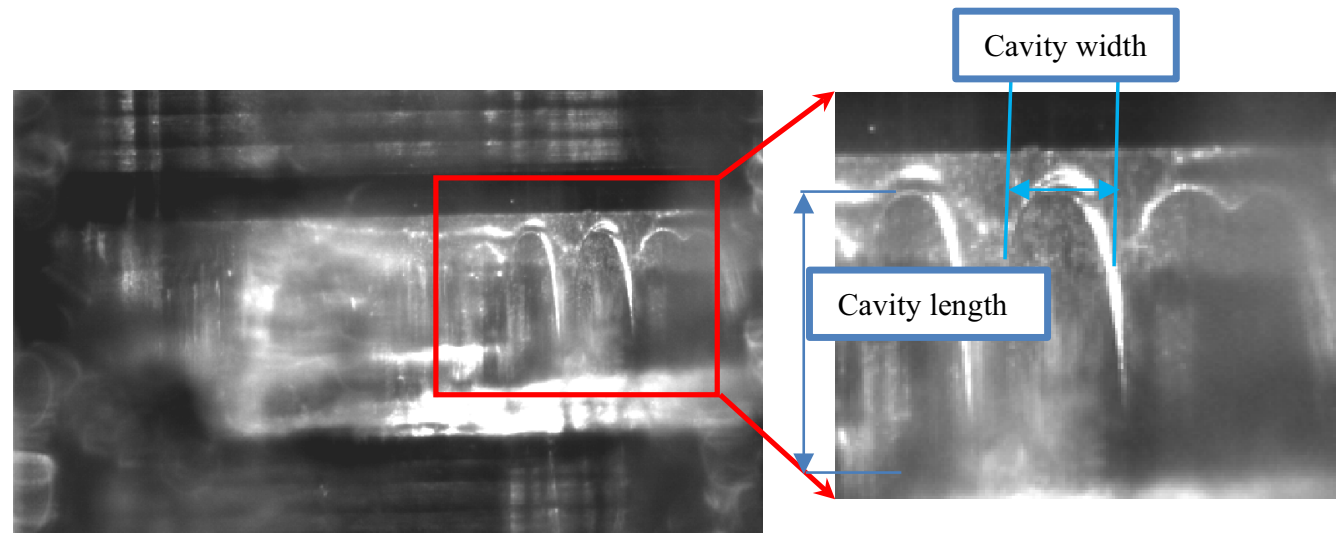

Fig. 3. Lister - Petter engine cavitation imaging and cavitation factor measurements $[7,11]$

\subsubsection{Hydra engine cavitation results}

The Ricardo-HYDRA engine test rig was fitted with a purpose-built visualisation window as in the case of the Lister-Petter engine. In this case, firing tests could be conducted $[5,6]$.

Cavitation is visualised through the quartz liner windows:

- video images captured with two high speed cameras coupled with three ARRI high intensity light sources were analysed.

- The images were post-processed with the aid of a custom-built algorithm.

The basic function of this algorithm is the extraction of matrices with elements of cavity length, cavity width, area of cavitation and number of cavities present under the piston-ring and the cylinder liner [5]. In Figure 4 side by side comparison is attempted for the test rig and engine visualisation results at different parts of the stroke.
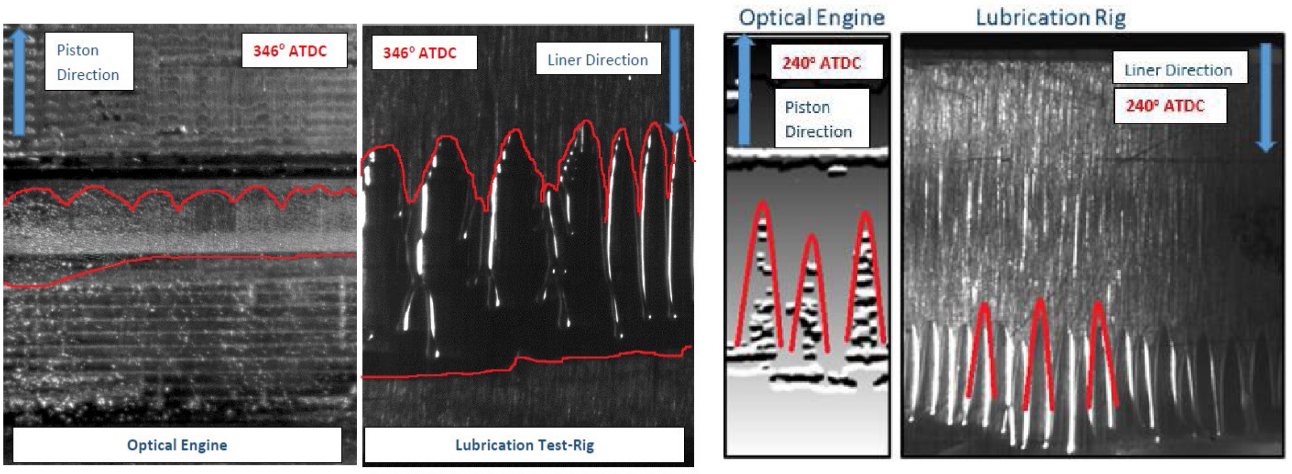

Fig. 4. Cavitation Comparison: Side by side optical engine and lubrication test rig cavity comparison a) At 346 ATDC and b) at 240 ATDC [5].

The different lubrication regimes encountered during the piston-ring motion are identified as: a) The friction force at contact (dead centers), due to asperity interaction: the lubrication regime is boundary. b) Next to the boundary lubrication region, there is a mixed region where the frictional force due to asperity interaction is much larger than the viscous losses. c) The ring makes a transition from mixed to full film lubrication and the hydrodynamic region where all frictional losses are due to viscous drag, resulting in a point of minimum $\mathrm{C}_{\mathrm{f}}$ (friction coefficient). As shown before with the help of extensive parametric 
study, the measured friction spikes are dependent upon piston-ring curvature, speed, load and temperature conditions and high temperature high shear (HTHS) viscosity of the lubricant [1]. The friction results should be combined with the cavitation imaging results, to perform analysis on the initiation of cavition, the squeeze film effect alteration due to speed, load and viscosity change and the area of cavitation as it is produced from the imaging.

\section{Discussion}

An investigation into frictional losses at the piston-ring cylinder liner contact must take into account the transitions from a state of full film lubrication to boundary lubrication [7]. Cavitation at the dead centers, with the formation of both a new cavitating region and the one that diminishes, leads to thinner lubricating films and higher friction forces. The saviour in this case is the squeeze film development, that in turn, alters its position under different operating conditions. There are extensive cavitating regions which contribute no radial support but generate frictional losses [7]. Fluid properties play the primary role in the formation and collapse of cavities (cavitation behaviour) while simultaneously influence cavitation behaviour directly by introducing changes in the film thickness [7, 13].

What should be noticed in the case of the parametric oil film pressure measurements for each piston - ring specimen, is that for the load variation tests the pressure peaks appear more uniform. Cavitation generation is largely dependent on pressure fluctuation.

\subsection{Lubricant Physical and Chemical Properties, Cavitation and Wear}

There is a possible link between the way lubricants cavitate and kinematic viscosity as results from imaging and friction in the simulating test rig have shown. One reliable way to extract and correlate with the formulation of each lubricant is the length of the cavity [5]. Formulation of the lubricants used in the parametric study affect at a great degree the initiation and the end of each cavitational phenomenon with the physical property of kinematic viscosity of the lubricant being a very good indicator.

At the same time, a lubricant without viscous modifier develops longer cavities, while a kinematic viscosity of greater value will offer a lower area of cavitation [5].

A parametric study test matrix with a wide range of lubricants of different properties, offers a collective view of how different formulations affect the behaviour and initiation of cavitation. Cavitation initiation points are varied according to speed, load and temperature testing [4]. Different forms of cavitation stages were identified close to the dead centers and then towards mid-stroke and accompanied speed and load testing. These different forms of cavitation - their shape and size (length and width) are dictated from reciprocating speed and viscosity of the lubricant as well as testing temperature which holds a detrimental effect on viscosity.

Cavitation damage results mainly from liquid impact on the bearing wall during cavity collapse. Since viscosity inhibits cavity growth and collapse, it is expected that the collapse velocity will be lower in a Newtonian lubricant than in a shear thinning lubricant. Shear thinning influences cavitation behaviour primarily by modifying the film thickness $[7,8,13]$. The shear thinning feature of multigrade lubricants and use of thinner monograde oils can generally reduce frictional losses but this may result in an increased wear of the cylinder liner [7]. The effect of film thickness on cavitation behaviour must also be taken into account while assessing the influence of viscosity and cavitation damage [8].

At boundary lubrication, the squeeze film effect prevails. Lower viscosity oils reduce frictional losses and increase efficiency but high volatility leads to hydrocarbon emissions [7].

The cavitation patterns are connected to the LIF measurements: it was shown that it can be either lubricant, bubble cavity or a mixture of both [12]. The cavitation patterns change 
for different testing conditions and chemical formulation as MOFT contributes to cavitation behaviour. For similar MOFT, the chemistry and resulting properties affect cavitation behaviour.

In terms of number of cavities, these are influenced by speed, load and temperature. It is therefore important to study the effect of oil properties and their influence on cavitation, its area and size of the microscopic forms. Observations of chemical formulation can lead to innovative additive designs and new properties and thus, improve efficiency for future research.

It is, of course, a matter of great importance to further investigate lubricant properties and friction and at a second stage cavitation and squeeze film development, as there is always a trade-off between performance and emissions control [8].

A textured surface can overcome the problem of changing the friction properties of the ring-liner interface. The grooves that contribute to the surface texture can be in either side, (ring or liner) and studies showed the existence of microcavitation on these grooves [7].

\section{Conclusions}

Cavitation causes the effective load area to shrink and the load capacity declines, even if specific hydrodynamic pressures remain high [7]. Different lubricants and variation of piston-rings geometry should be considered. Squeeze film is altered due to speed, load, temperature and high temperature high shear viscosity and at the same time, with its collapsion close to the dead center of the stroke, friction peaks appear. They provide indications of wear development at the piston assembly interface [7]. Cavitation patterns are connected to the LIF probe reading and the probe reads an area that is either lubricant, bubble cavity or mixture of both cavity and lubricant. The change of cavitation pattern is stressed out for the different testing conditions and the chemical formulation of the lubricant.

The lubrication regime properties are altered at the areas of interest, producing different results in terms of cavitation initiation, its area and along with the friction measurements the overall or local (close to the dead center) friction power losses. This eventually has an effect on the pressure profile in the entrainment direction, cavitation initiation and pattern and consequently the ring-pack's load carrying capacity.

\section{References}

1. P. Dellis, Effect of friction force between piston rings and liner: a parametric study of speed, load, temperature, piston-ring curvature, and high-temperature, high-shear viscosity, Proc IMechE, Part J: J Engin. Trib.; 224(5): 411-426, (2010).

2. P. Dellis, and C. Arcoumanis, Cavitation development in the lubricant film of a reciprocating piston-ring assembly, Proc. IMechE, Part J: J. Engin. Trib., 218, 157171. DOI: $10.1243 / 1350650041323340$, (2004).

3. P. Dellis, Aspects of lubrication in piston cylinder assemblies, $(\mathrm{PhD}$ Thesis, Mech.Eng. Dept., Imperial College London, 2005).

4. P. Dellis, Laser-induced fluorescence measurements in a single ring test rig: Evidence of cavitation and the effect of different operating conditions and lubricants in cavitation patterns and initiation, Int. J. Engine Res., 21(9): 1597-1611, DOI:10.1177/1468087418819254, (2019).

5. I. Vasilakos, Cavitation in the cylinder-liner and piston-ring interaction in internal combustion engines, (Doctoral Thesis, School of Engin. and Mathem. Sc., City University London, London, 2017). 
6. J. M. Nouri, I. Vasilakos and Y. Yan, Cavitation between cylinder-liner and piston-ring in a new designed optical IC engine, Int. J. Engine Res., first published online: April 9, 2021.

7. P. S. Dellis, Piston-ring performance: limitations from cavitation and friction, Intern. Journal of Structural Integrity, 10(3): 304-324, DOI:10.1108/IJSI-09-2018-0053, (2019).

8. P. Dellis, The effect of friction force and cavitation on wear in piston-cylinder assemblies, MATEC Web of Conferences 188, 04012, DOI: 10.1051/matecconf/201818804012, (2018).

9. P. Dellis, Cavitation in engine lubricants: visualisation experiments in both a single ring test rig and a single cylinder motored diesel engine to complement on the theoretical modeling of cavitation, ASME Proceedings, 10th International Symposium on Cavitation - CAV2018 Baltimore, Maryland, USA, May 14 - 16, 206-210, CAV1805042, (2018).

10. P. Ostovar, Fluid aspects of piston-ring lubrication, (PhD Thesis, Dept. of Mech. Eng., Imperial College London, London, 1996).

11. P. Dellis, Aspects of Lubrication in a Reciprocating Single-ring Test Rig and Further Implementation to Engine Applications, Trib. in Ind., 39 No. 2, 260-269, DOI: 10.24874/ti.2017.39.02.14, (2017).

12. P. Dellis, A parametric study on oil film pressure measurements in a single piston-ring configuration, Int. J. of Engine Res., 14(2), 122-137, DOI: 10.1177/1468087412440540, (2013).

13. A. Rastogi, and R. K. Gupta, Effect of shear thinning on cavitation in lubricant films. SAE paper 922286, (1986). 